\title{
Fabry-Perot filter based solar video magnetograph
}

\author{
S.K. Mathew, A. Bhatnagar, C.D. Prasad, and A. Ambastha \\ Udaipur Solar Observatory, Physical Research Laboratory, Udaipur - 313001, India \\ e-mail: shibu@uso.ernet.in, arvind@uso.ernet.in, debi@uso.ernet.in, ashok@uso.ernet.in
}

Received April 20; accepted June 11, 1998

\begin{abstract}
A tunable Lithium Niobate $\left(\mathrm{LiNbO}_{3}\right)$ FabryPerot filter (FP) (passband $165 \mathrm{~m} \AA$ at $6122 \AA$ ) based video magnetograph has been designed and fabricated. This instrument is capable of providing near simultaneous observations of photospheric longitudinal magnetic field, chromospheric $\mathrm{H} \alpha$, and photospheric $\mathrm{CaI}$ pictures using the same telescope and back-end set-up. The magnetic field measurements are made by using the polarization properties of the Zeeman components of the photospheric CaI line at $6122 \AA$ (Landé $g$ factor of 1.75). The CaI line has been chosen due to its low temperature sensitivity and no blend with other solar or atmospheric lines. A variable electro-optic quarter wave retarder, $\mathrm{KD}^{*} \mathrm{P}$ (Potassium diDeuterium Phosphate) along with a linear polarizer is used for analyzing the circular polarization of the Zeeman components. The filter tuned at $140 \mathrm{~m} \AA$ away from the line center in the blue wing is found to give the best linear response for the field strength up to 1500 Gauss. A field of view (FOV) of $\sim 4 \times 3 \mathrm{arcmin}$ on the solar disk is imaged using a $699 \times 288$ pixel Cohu CCD camera in synchronous with the $\mathrm{KD}^{*} \mathrm{P}$ modulation. The $\lambda / 4$ modulation is achieved by applying \pm 2100 volts to the $\mathrm{KD}^{*} \mathrm{P}$ to obtain alternate frames of oppositely circular polarized images. These images are stored in separate frame buffers of an image acquisition system. To achieve high signal to noise ratio, a large number of images (maximum 256) are added in the respective frame buffers and then the difference between the left and the right circularly polarized images is obtained. This difference is related to the magnetic field strength. On comparing the video magnetograms (VMG) obtained at Udaipur Solar Observatory (USO) on 09 April 1997 at 09:32 UT with those taken by SOHO/MDI at 09:41 UT, it was found that all the magnetic features matched very well in both the magnetograms. In this paper we present the details of the instrument and examples of observations.
\end{abstract}

Send offprint requests to: S.K. Mathew
Key words: instruments: polarimeter - Sun: activity; flares; magnetic fields

\section{Introduction}

A filter magnetograph is the more suitable instrument for observing rapid changes in the magnetic fields, associated with the solar dynamic phenomena compared to the spectrograph based magnetographs (Zirin 1995). The pre-flare syndrome and the flare activity is best observed by using the chromospheric $\mathrm{H} \alpha$ line. The emergence of new magnetic flux is believed to be important for triggering most of the large solar flares (Heyvaerts et al. 1977; Canfield et al. 1993; Debi et al. 1998). These fluxes appear as pores and bipolar sunspots in photoheliograms and arch filament system (AFS) in H $\alpha$ filtergrams. Therefore it is important to have simultaneous or near simultaneous observations of the magnetic field, chromospheric and photospheric activities. The filter magnetographs operating at Marshall Space Flight center, Big Bear Solar Observatory (U.S.A.) and Huairou solar station (China) have shown the importance of these instruments in flare study. However, the birefringent filters used in these magnetographs can not be tuned rapidly to different wavelengths. Keeping in view of the above factors, we have designed and fabricated a voltage tunable $\mathrm{LiNbO}_{3}$ Fabry-Perot filter based video magnetograph, which can obtain near simultaneous photospheric magnetograms, $\mathrm{H} \alpha$ filtergrams and continuum images of an active region. Since these images are made by using the same imaging set-up, the problems of registration and inter calibration are minimized.

The Fabry-Perot (FP) etalons are extensively used in astronomy (Meaburn 1970; Vaughan 1989). The availability of high finesse FP etalons with large free spectral range made it possible to use them in solar observations (Rust et al. 1987; Bonaccini \& Stauffer 1990; Debi et al. 1998). Compared to the birefringent Lyot filters which are extensively used in solar observations, the $\mathrm{LiNbO}_{3} \mathrm{FP}$ etalons 
have the added advantage of fast voltage tunability which is very convenient for multi-wavelength observations.

The USO video magnetograph (VMG) is the only such instrument operational in the Indian longitude and fills an important gap for continuous coverage of solar magnetic field observations. The solar video magnetograph operating at USO could provide valuable data for synoptic studies of evolution of active regions. In this paper we present the details of the instrument and examples of observations.

\section{Basic principle of solar magnetograph}

The principle of Zeeman splitting of a spectral line, is employed to measure the solar magnetic field. In the case of longitudinal magnetic field the $\sigma_{1}$ and $\sigma_{2}$ components of the Zeeman triplet are left and right circularly polarized. For the transverse magnetic field the $\sigma_{1}$ and $\sigma_{2}$ components are linearly polarized parallel to the field and the unshifted $\pi$ component is linearly polarized perpendicular to the field. The $\sigma_{1}$ and $\sigma_{2}$ components are separated in wavelength from the unshifted line,

$\Delta \lambda_{H}=4.6710^{-13} \mathrm{~g} \lambda^{2} H$

where $H$ is the field strength in Gauss and $g$ is the Landé factor of the spectral line, $\lambda$ and $\Delta \lambda$ are in $\AA$ units. For weak magnetic fields, the direct measurement of Zeeman splitting is not possible even with very high resolution spectrographs. Therefore the polarization properties of the Zeeman components is used to measure the magnetic field. Since the $\sigma_{1}$ and $\sigma_{2}$ components are shifted in wavelengths and oppositely polarized, choosing the left or right polarized light i.e., $\sigma_{1}$ or $\sigma_{2}$, entering the system corresponds to shifting of the lines. This principle is used in our video magnetograph. We tune the narrow band filter on the blue wing of the magnetically sensitive CaI $6122 \AA$ line. It is found that tuning the filter at $140 \mathrm{m \AA}$ away from the line center gives acceptable linearity for magnetic field measurement up to \pm 1500 Gauss. By switching an electrooptic variable $\lambda / 4$ retarder, the field-of-view (FOV) of solar photosphere are imaged alternately in $\sigma_{1}$ and $\sigma_{2}$ components. A number of images, up to 256, are added for each $\sigma_{1}$ and $\sigma_{2}$ components separately in order to achieve the acceptable signal to noise ratio. The difference between the averaged $\sigma_{1}$ and $\sigma_{2}$ images is a measure of the longitudinal magnetic field.

\section{Instrumentation}

\subsection{Optical layout}

The optical layout of the video magnetograph along with the light feed is given in Fig. 1. The objective lens L1 $(\phi=15 \mathrm{~cm}, \mathrm{f} / 15)$ makes a solar image of $22 \mathrm{~mm}$ diameter at the focal plane where a field stop FS is placed. A heat filter $\mathbf{H F}$ is used to reduce the heat load on the optics and
CCD camera saturation by blocking the IR radiation. A portion of the image is enlarged by a factor of 2.7 by a relay lens $\mathbf{L} 2$ to yield a $60 \mathrm{~mm}$ solar image $(f / 40)$. The pre-filter wheel PFW, KD* $\mathbf{P}$ modulator, linear polarizer $\mathbf{L P}$ and $\mathbf{F P}$ etalon filter are placed in the telecentric beam following the lens L2. The fast axis of the $\mathbf{K D}^{*} \mathbf{P}$ crystal makes an angle of $45^{\circ}$ with the linear polarizer LP. Finally the image is recorded by a CCD camera. To avoid scattered light and ambient temperature variations, all the components are mounted on the optical bench, and are enclosed in a wooden box EN.

\subsection{Fabry-Perot narrow band filter}

A $60 \mathrm{~mm}$ aperture, high finesse voltage tunable $\mathrm{LiNbO}_{3}$ FP etalon acquired from CSIRO Australia, is used as a narrow band filter to isolate a portion at the wing of the selected absorption line. The FP etalon is made by using a $\mathrm{LiNbO}_{3}$ substrate of $0.175 \mathrm{~mm}$ thickness, both sides of which are polished to $\lambda / 200$ and coated with high reflective $\mathrm{SiO}_{2}$ and $\mathrm{Ta}_{2} \mathrm{O}_{5}$ films. The resultant etalon has a reflectivity of $93 \%$ over a wavelength range of 5000 to $6700 \AA$. The tunability of the etalon is achieved by applying high voltage across the $\mathrm{LiNbO}_{3}$ wafer which varies its refractive index. A conductive ITO (Indium Tin Oxide) coating is deposited for the application of electric field across the crystal. The high voltage terminals made of gold wires are bonded to the ITO coating with the help of silver epoxy. The details of the FP parameters are given in Table 1. In order to avoid the drift of the FP band pass due to a change in the ambient temperature, the etalon is enclosed in a constant temperature oven. The oven temperature is maintained at $43{ }^{\circ} \mathrm{C}$ with a stability of $\pm 0.05{ }^{\circ} \mathrm{C}$ which provides a wavelength stability $5 \mathrm{m \AA}$. A bench test was performed for determining different parameters of the FP etalon, by placing it in front of the USO Littrow spectrograph coupled with $15 \mathrm{~cm}$ Zeiss coudé telescope. This spectrograph has a dispersion of $0.047 \AA /$ pixel and an $f$-ratio of $f / 40$ similar to the light beam used for the video magnetograph. Figure 2a show the observed FP channel spectrum recorded near CaI $6122 \AA$. The observed profiles are corrected for the spectrograph instrumental broadening, using the CaI $6122 \AA$ line profile obtained from KPNO digital solar spectral atlas (Debi et al. 1998). The corrected profile is shown in Fig. 2b. At $\mathrm{H} \alpha 6563 \AA$ also, the results are similar. Solar spectra were recorded by removing the FP etalon placed in front of the spectrograph slit. The measured Full-width at half maximum (FWHM), Free-Spectral range (FSR), and finesse (FSR/FWHM) of the etalon are given in the Table 1 . The voltage tunability of the etalon was determined by measuring the wavelength shift of the channel spectrum as a function of voltage in the range of -3000 to +3000 Volts in steps of 50 Volts. With in this range the wavelength shift is linear with voltage and is found to $0.45 \AA$ per 1000 Volts. 


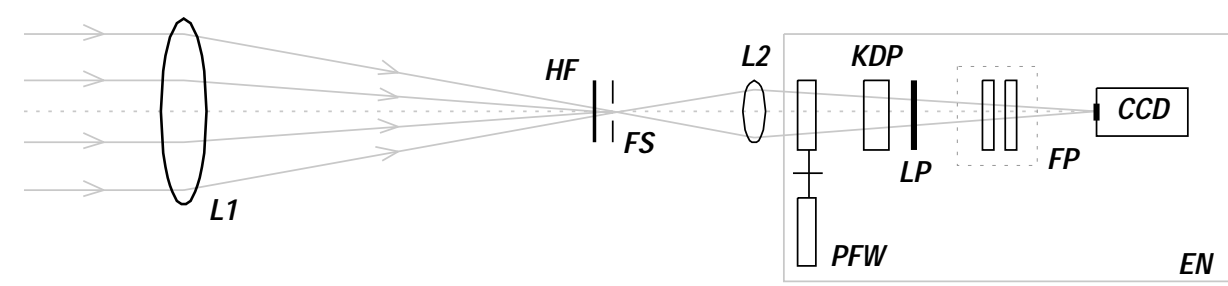

Fig. 1. Optical layout of USO video magnetograph. L1 - $15 \mathrm{~cm}$ objective lens, HF - heat filter, FS - field stop, L2 - relay lens, PFW - pre-filter wheel, KDP - KD*P electro-optic modulator, LP - linear polarizer, FP - Fabry-Perot etalon filter, CCD - CCD video camera, EN - Wooden enclosure
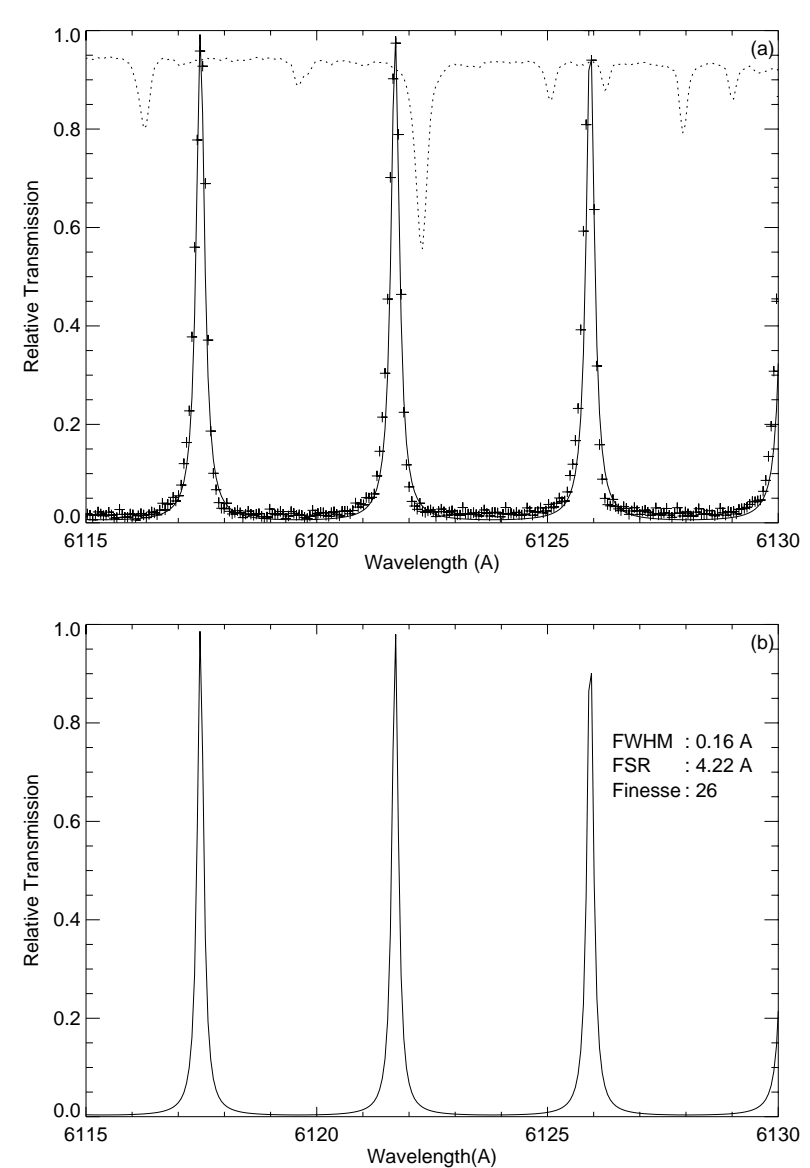

Fig. 2. The observed and corrected Fabry-Perot channel spectra: a) and b) show the observed and corrected channel spectra at $6122 \AA$. The dotted line shows the solar spectrum, the intensity is plotted in arbitrary units

To isolate $6122 \AA(\mathrm{CaI})$ and $6563 \AA(\mathrm{H} \alpha)$ lines two narrow band interference filters of passband $2.6 \AA$ and $3.5 \AA$ respectively are placed before the FP, and the voltage on the FP is accordingly changed to obtain VMG in $6122 \AA$ line and $\mathrm{H} \alpha$ filtergrams. These two pre-filters are also enclosed in separate temperature controlled ovens and mounted on computer controlled filter wheel, in order to make near simultaneous magnetic field, chromospheric, and photospheric observations.
Table 1. Narrow band filter parameters

$$
\begin{array}{ll}
\hline \text { Etalon Properties } & \\
\hline \text { Substrate material } & : \text { Z-cut } \mathrm{LiNbO}_{3} \\
\text { Substrate diameter } & : 73.6 \mathrm{~mm} \\
\text { Usable aperture } & : 60 \mathrm{~mm} \\
\text { Etalon thickness } & : 0.175 \mathrm{~mm} \\
\text { Coating } & : \text { Multilayer broad band, hard } \\
& \text { ion assisted deposition } \\
& \text { of } \mathrm{SiO}_{2}, \mathrm{Ta}_{2} \mathrm{O}_{5}, \text { ITO } \\
\text { Reflectivity } & : 0.95 \text { at } \lambda=6120 \AA \\
\text { Voltage sensitivity } & : 0.45 \AA \text { per } 1000 \text { Volts }
\end{array}
$$

\begin{tabular}{|c|c|c|}
\hline Free Spectral Range (̊) & 4.22 & 5.05 \\
\hline Passband $(\AA)$ & 0.16 & 0.22 \\
\hline Finesse (FSR/FWHM) & 26 & 23 \\
\hline Voltage applied (Volts) & -1200 & +2500 \\
\hline \multicolumn{3}{|l|}{ Pre-filter parameters } \\
\hline Central wavelength $(\AA)$ & 6122.23 & 6563.98 \\
\hline Bandwidth $(\AA)$ & 2.68 & 3.58 \\
\hline Peak transmission (\%) & 33.50 & 23.03 \\
\hline Ambient temperature $\left({ }^{\circ} \mathrm{C}\right)$ & 43.0 & 43.0 \\
\hline
\end{tabular}

\begin{tabular}{ll}
\hline FP filter parameters & CaI $6122 \AA \mathrm{A} \mathrm{H} \alpha 6563 \mathrm{~A}$ \\
\hline
\end{tabular}

\subsection{Circular polarization analyzer}

A $\mathrm{KD}^{*} \mathrm{P}$ electro-optic quarter wave plate and a linear polarizer constitute the circular polarization analyzer for measuring the longitudinal magnetic field. The modulator uses a thin $(<3 \mathrm{~mm})$ Meadowlark $\mathrm{KD}^{*} \mathrm{P}$ crystal which makes these cells suitable for using them in convergent light beam slower than $f / 20$ (West 1989). The fast axis of the $\mathrm{KD}^{*} \mathrm{P}$ crystal is aligned at $45^{\circ}$ to the transmission axis of the linear polarizer and housed in an insulated enclosure. The $\mathrm{KD}^{*} \mathrm{P}$ crystal is converted to $\pm \lambda / 4$ retarders (at $\lambda=6122 \AA$ ) by applying \pm 2100 Volts. In order to operate the $\mathrm{KD}^{*} \mathrm{P}$ modulator a fast switchable high voltage power supply was made, which can provide \pm 2100 Volts on the application of low voltage TTL pulses at the input. 
The TTL pulses are obtained from the centronics port of the image acquisition system and synchronized with the image frame acquisition, such that alternately captured video frames contain left or right circularly polarized images. The left and right circular Zeeman components are converted in to two mutually perpendicular linear polarizations depending on the sign of the applied voltage. One of the components is blocked by the linear polarizer allowing the selection of the image corresponding to left or right circular polarization in the emerging beam.

\subsection{Detector - Cohu 4710 series $C C D$ camera}

The detector used in the video magnetograph is a Cohu make CCD monochrome camera with image sensor chip TC277 from Texas Instruments. This camera provides high resolution images with sensitivity as low as 0.25 lux, zero geometric distortion and no lag or retention of images. The CCD used is a frame transfer device with $9.2 \times 16.8 \mu \mathrm{m}$ pixels arranged in $699 \times 576$ array in which half of the pixel rows are masked for image storage and the other half are exposed to light. This makes a resultant image area of $6.4 \times 4.8 \mathrm{~mm}$ on the CCD chip. CCIR scanning system is employed for the image read out, where a single video field (one video frame contains two video fields -odd and even) takes $1 / 50$ seconds for scanning. During every video field the charge accumulated in the storage section is read out while the image section is exposed. The vertical blanking pulses after each video field (two vertical blanking pulses for each video frame) in the CCIR video output is detected through software and used for the synchronization of the $\mathrm{KD}^{*} \mathrm{P}$ switching and the entire data acquisition and reduction process. In the present optical set-up an area of $\sim 4 \times 3$ arcmin of the solar disk is imaged by the camera, which gives a resolution of $0.3 \times 0.6 \mathrm{arcsec} / \mathrm{pixel}$.

\subsection{Image acquisition and control system}

Block diagram of the image acquisition and control system of the video magnetograph is given in Fig. 3. An Innovision Inc., workstation, based on Motorola, MC68030, single board VMEbus computer, and integrated with Imaging Technology Series 150 image processing modules makes the complete data acquisition and control system. The image processing modules consists of one analogue-todigital interface (ADI) unit, two frame buffers (FB) and one arithmetic and logic unit (ALU); all connected to the VMEbus of the host computer. The combination of the host computer, series 150 modules and suitable software can perform complex on-line real time digital image processing tasks such as averaging of images and subtraction. The ADI employs an 8-bit flash A/D converter at $10 \mathrm{MHz}$ sampling rate, which digitizes the video input signal to 256 gray levels. The video bus transmits the digitized data (VDI) to all the other modules. The frame

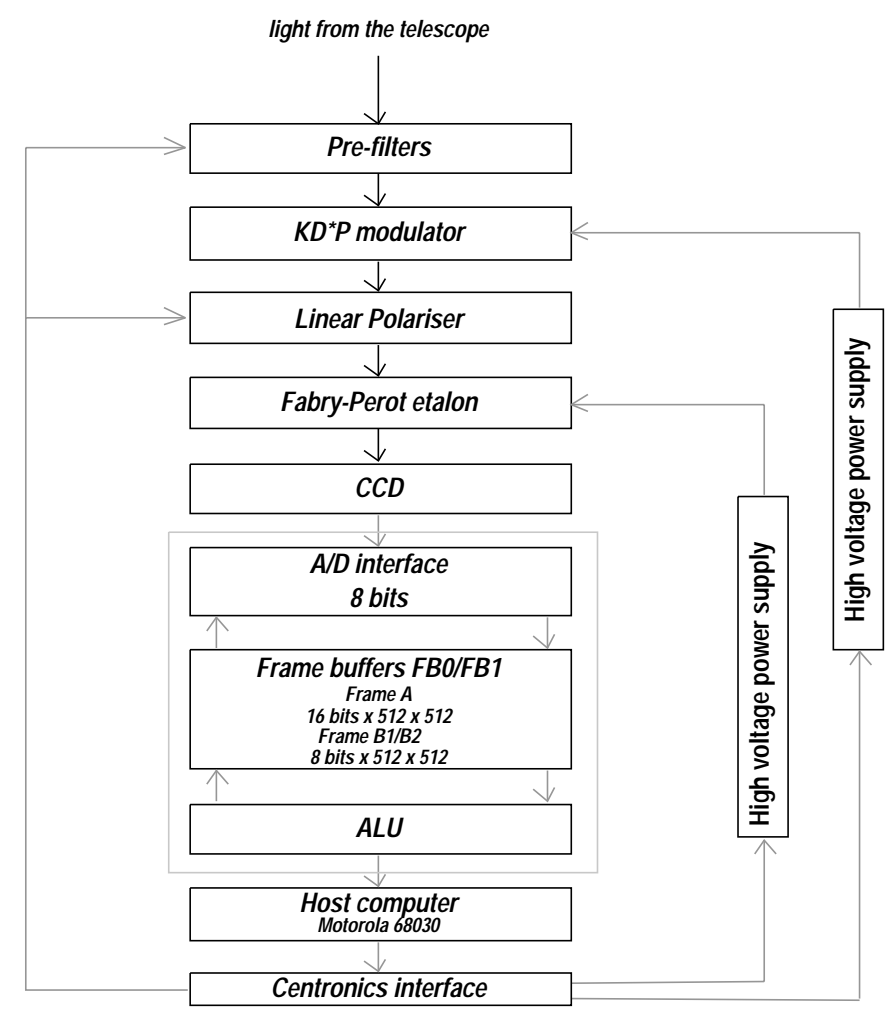

Fig. 3. The schematic diagram of the optics, image acquisition system and control electronics

buffers FB0 and FB1 contains the image storage required for the real time processing of the data. Each FB consists of a single 512 by 512 by 16-bit frame store (FRAME A) and two 512 by 512 by 8-bit frame stores (B1 and B2). ALU is a pipelined image processor which provides real time image processing capabilities when used with ADI and FB. The images from FRAME A and FRAME B are processed by ALU and the result is stored in FRAME A, which is finally transferred to the host computer.

A software was developed using C-language and ITEX 150/151 image processing library (Imaging Tech, Inc) for real time processing and control of the video magnetograph (Mathew 1998). The flow chart illustrates the sequence of operation performed by the software during a single acquisition cycle (Fig. 4a). The centronics port data bits D0 to D5, performs various control operations such as opening and closing the telescope shutter, changing the pre-filter, moving the relay lens for focusing the image for $\mathrm{H} \alpha$ and $\mathrm{CaI}$, changing the polarizer wheel, and switching the $\mathrm{KD}^{*} \mathrm{P}$ high voltage supply. A single acquisition cycle consists of several operations to obtain selected number of magnetograms, CaI $6122 \AA$ and $\mathrm{H} \alpha$ images. To obtain the photospheric CaI $6122 \AA$ images, the filter is kept tuned at the same position where the Stokes $V$ signal is taken. The flow chart in Fig. 4b shows various steps involved to obtain a single video magnetogram. For making magnetograms, the Series150 modules are set up for continuous acquisition and adding up of the images. The ALU is programmed 
(a)

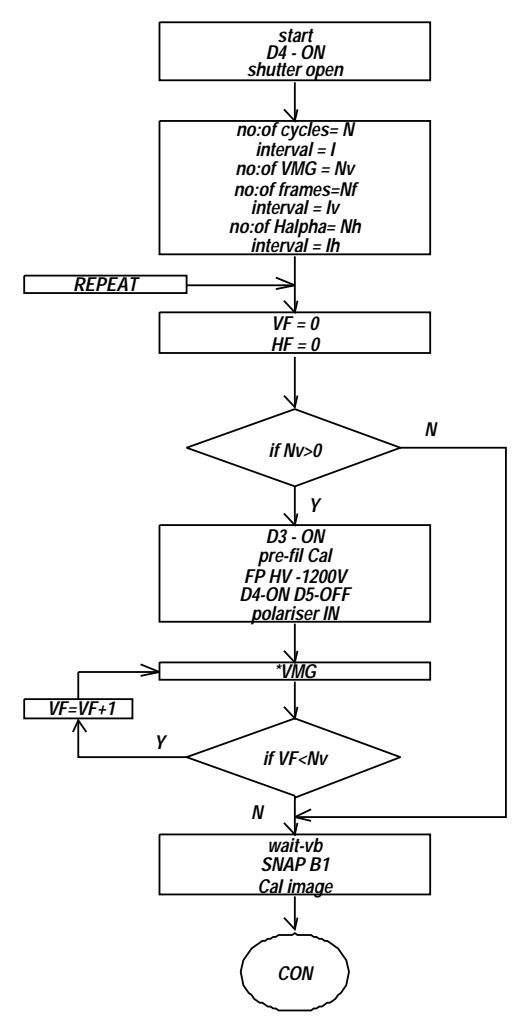

(b)

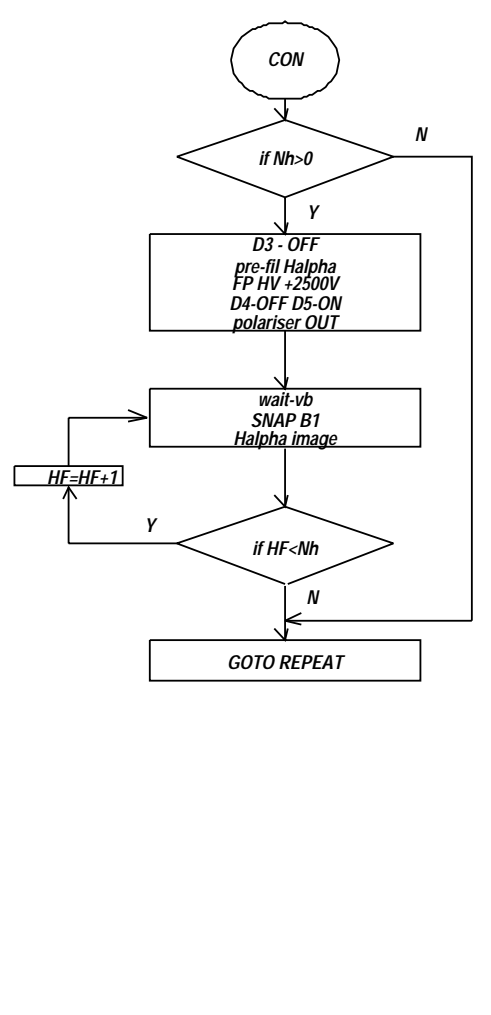

${ }^{*} V M G$

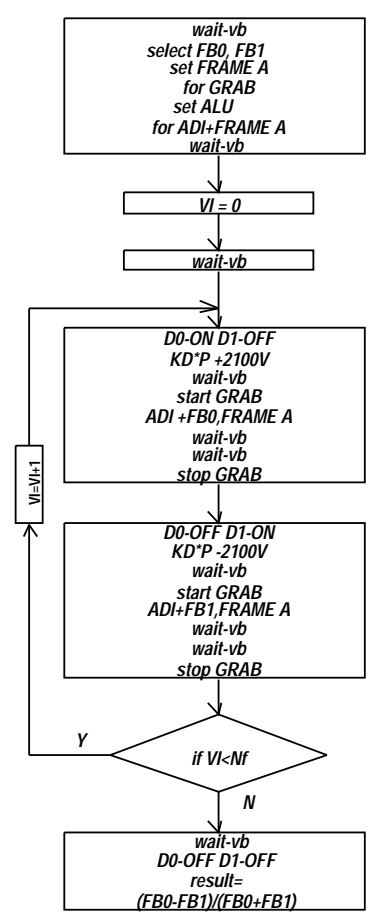

Fig. 4. Flow chart showing: a) the operations involved in a single cycle of image acquisition, b) VMG operation for a single video magnetogram

for adding the incoming video data on to the pervious image already present in the FRAME A. The alternate incoming video images are routed to FRAME A of FB0 and FB1 respectively. This is synchronized with the $\mathrm{KD}^{*} \mathrm{P}$ high voltage switching such that the alternate frames are taken in the left and right circular polarizations. This process can be repeated for a pre-selected number of frames (maximum of 256 frames) to increase the signal to noise $(\mathrm{S} / \mathrm{N})$ ratio. To integrate 256 frames, the systems takes 12 seconds. The whole sequence of one observation, takes about 1 minute which consists of a video magnetogram averaged over 256 frames, CaI photospheric image and $\mathrm{H} \alpha$ filtergram.

\subsection{Calibration and comparison}

The calibration of the video magnetograms were made by using the profile line slope method (Varsik 1995), which is based on the weak field approximation (Jefferies \& Mickey 1991). The Stokes parameter $V$ can be expressed as,

$B_{\|}=k_{\text {cal }} V$

$k_{\text {cal }}=\left(-\frac{\mathrm{d} I}{\mathrm{~d} \lambda} \mu \lambda_{0}^{2} g\right)^{-1}$ where $\mu=e / 4 \pi \mathrm{mc}^{2} ; e$ and $m$ are the charge and mass of the electron, $\lambda_{0}$ the central wavelength of observation in $\AA, g$ the Landé factor of transition for absorption line, $B_{\|}$is the longitudinal magnetic field in Gauss and $k_{\text {cal }}$ is the calibration factor. $I$ the Stokes intensity obtained in the absence of magnetic field and $V$ is the intensity obtained by subtracting a single pair of video frames taken in opposite circular polarization. $\mathrm{d} I / \mathrm{d} \lambda$ is the measured line slope by tuning the filter from $-40 \mathrm{~m} \AA$ to $-170 \mathrm{~m} \AA$ from the line center in the steps of $25 \mathrm{~m} \AA$. The measured slope $\mathrm{d} I / \mathrm{d} \lambda$ is found to be 0.047 , which gives the value of $k_{\text {cal }}=2059$ using Eq. (3). This constant is used in Eq. (2) for conversion of measured Stokes $V$ intensity into the magnetic field values in Gauss. The measured longitudinal magnetic field is the observed average field over the spatial resolution element and limited by the solar seeing. Further as the spectral resolution of the FP etalon is small, the accuracy of calibration by this method is rather low.

Figures $5 \mathrm{a}$ and $\mathrm{b}$ show USO and SOHO/MDI video magnetograms obtained around the same time on 09 April 1997. The SOHO/MDI magnetogram is a part of the calibrated full-disk data (Scherrer et al. 1995). It may be noticed that all the features observed in both the video magnetograms match well. After accurate registration of 


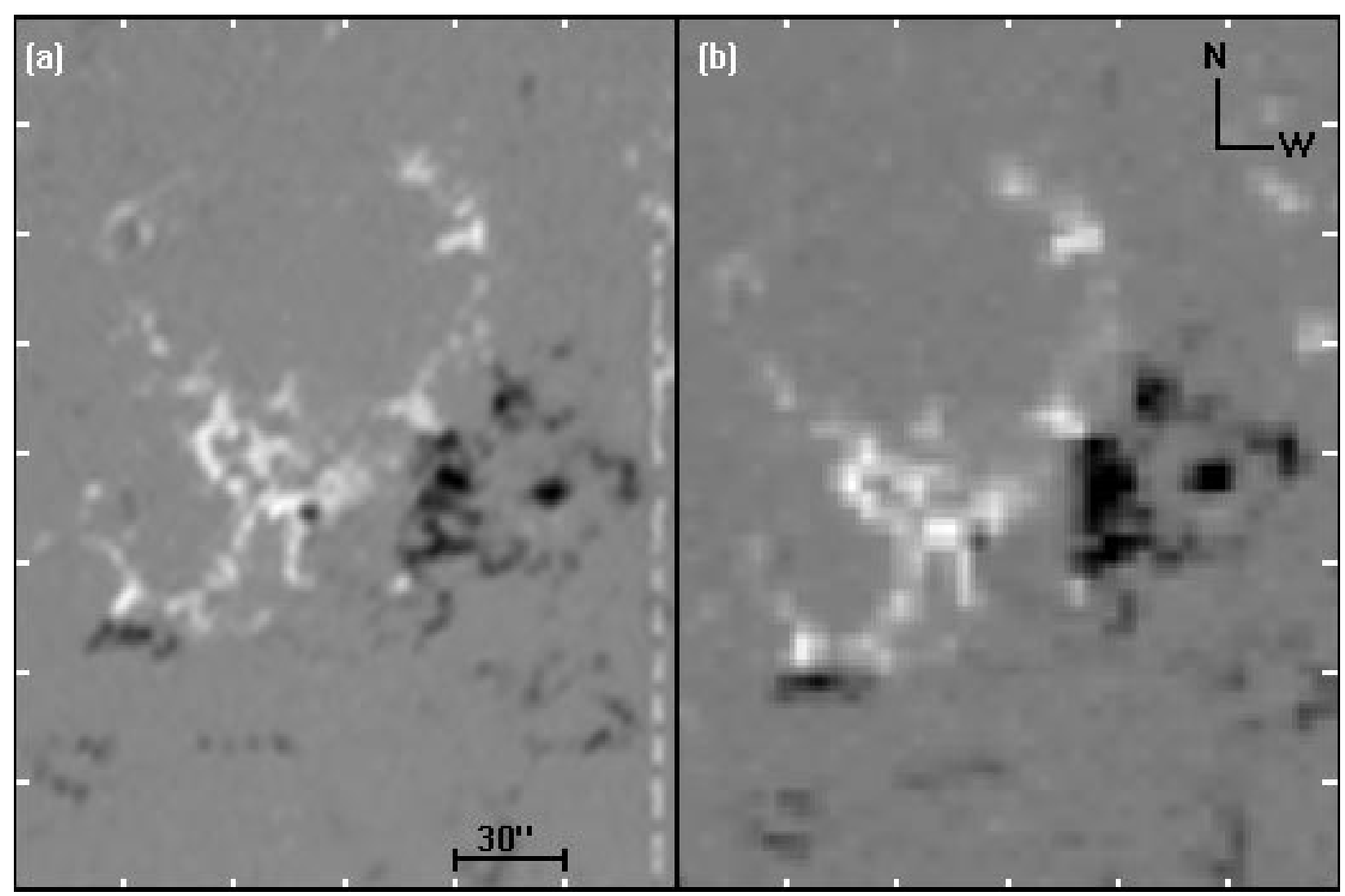

Fig. 5. The magnetograms obtained for comparison on 09 April 1997 by: a) USO video magnetograph at 09:32 UT, b) SOHO/MDI at 09:41 UT

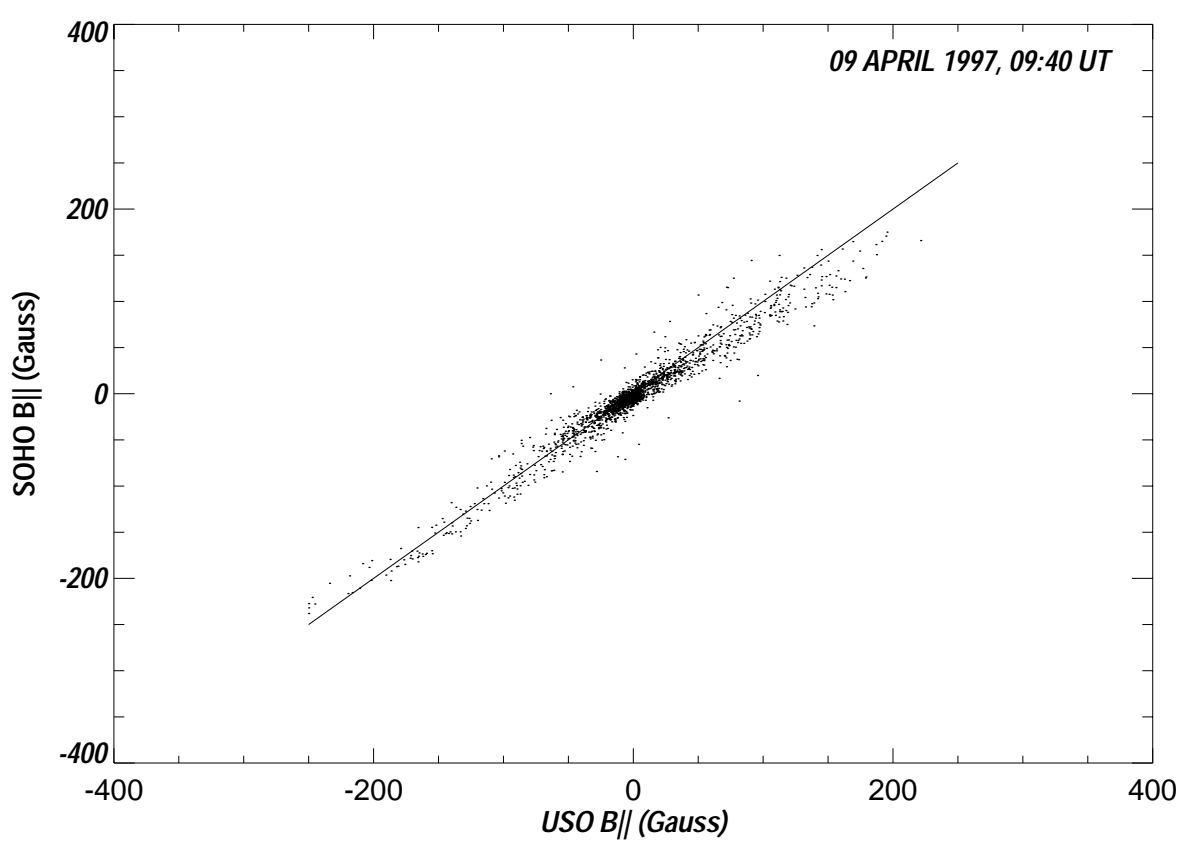

Fig. 6. Scatter plot made between calibrated USO and SOHO magnetograms 


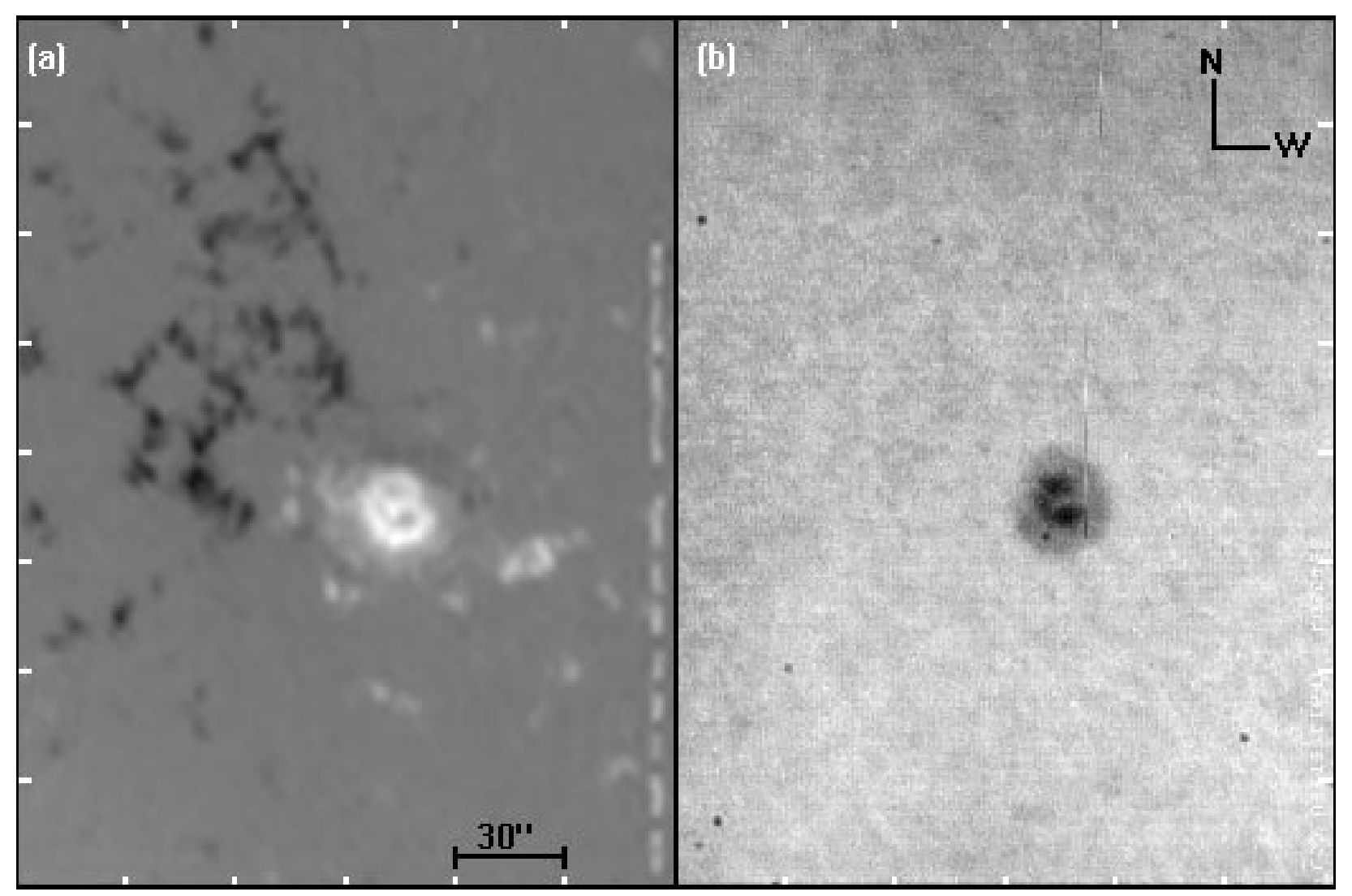

Fig. 7. A typical: a) video magnetogram and b) CaI $6122 \AA(-140 \mathrm{~m} \AA)$ image

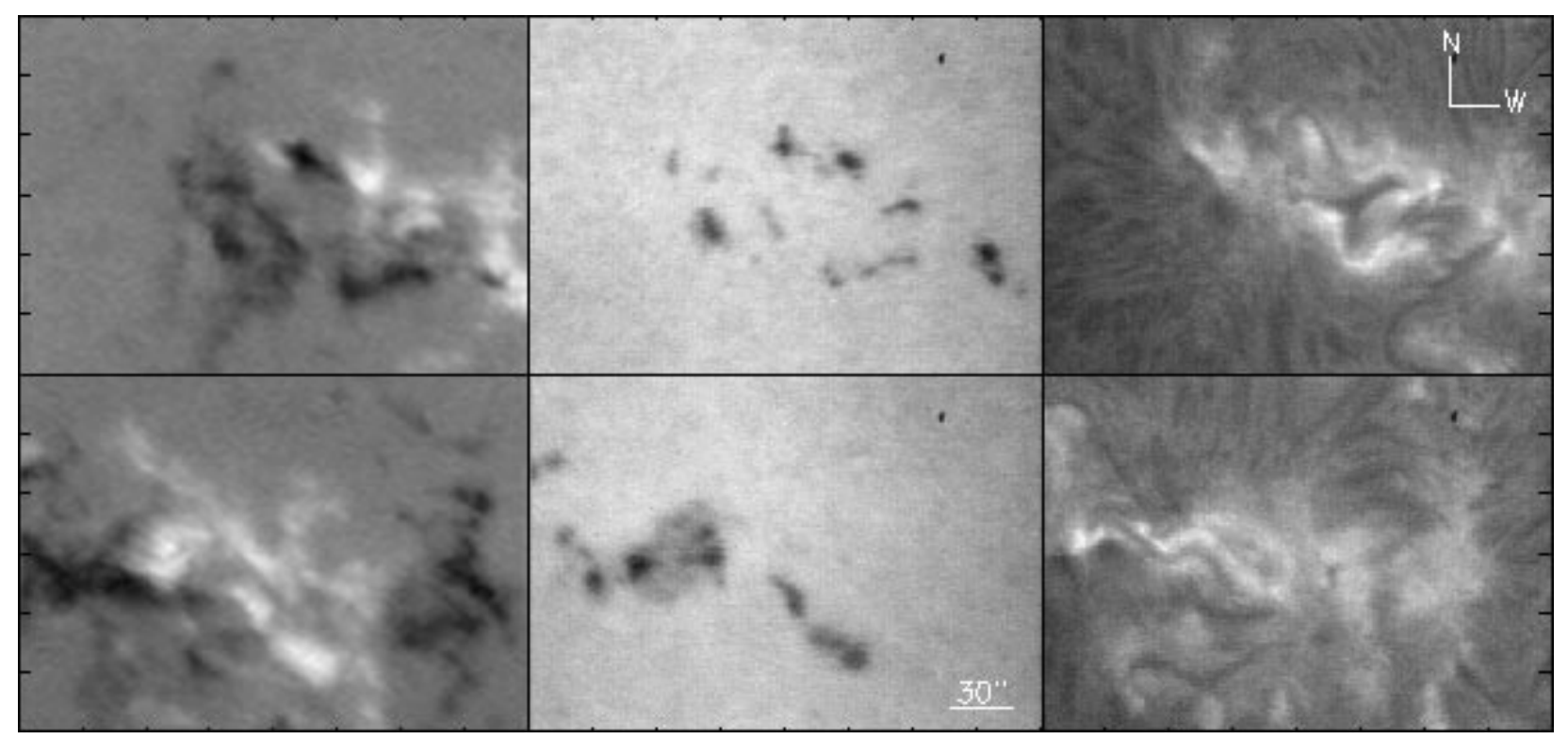

Fig. 8. Composite picture of magnetogram, CaI $6122 \AA(-140 \mathrm{~m} \AA)$ and $\mathrm{H} \alpha$ images showing one cycle multi-wavelength observation. The upper panel shows the images of active region NOAA\#7843 taken on 19 February 1995 and the lower panel shows the same active region on 20 February 1995 
the two VMGs and degrading the spatial resolution of USO VMG to match the SOHO/MDI, a scatter diagram has been plotted and shown in Fig. 6. This plot demonstrate the validity of the calibration procedure, except for a small deviation of the slope of the scatter line from unity. This deviation may be attributed to the use of different spectral lines and difference in spectral and spatial resolution of the two instruments.

\section{Example of observations}

The USO video magnetograph is being used for nearsimultaneous observations of longitudinal magnetic field, photospheric, and $\mathrm{H} \alpha$ chromospheric activity. Figure 7 shows an example of a typical video magnetogram and the corresponding image in the blue wing of CaI $6122 \AA$ line. During the best seeing conditions, the fine magnetic features of about $2-4$ arcsec could be observed. The minimum detectable field was found to be 15 Gauss as obtained from the background noise level. Figure 8 shows an example of video magnetogram, $\mathrm{CaI} 6122 \AA$ and $\mathrm{H} \alpha$ images of active region NOAA 7843 obtained on 19 and 20 February 1995 in a single cycle of operation.

\section{Summary}

A video magnetograph using a voltage tunable $\mathrm{LiNbO}_{3}$ Fabry-Perot etalon has been designed and constructed and is operational at Udaipur Solar Observatory. The instrument is used in $f / 40$ beam of the telescope with an objective aperture of $15 \mathrm{~cm}$ and an imaging relay lens in telecentric mode. The comparison of USO magnetograms with $\mathrm{SOHO} / \mathrm{MDI}$ data shows the consistency of our measurements. The preliminary data show the capability of the instrument to take high spatial and temporal resolution observations which can be used to undertake study of various problems related to the magnetic field and flare productivity in active regions. This instrument is the first such facility in the Indian longitudes and fills an important gap for continuous coverage of observations of solar active regions.
Acknowledgements. Our thanks are due to Prof. F. Kneer for his valuable comments. The financial support for the present work comes from the Department of Space (DOS), Government of India and Indo-US scientific collaboration (NSF Grant INT - 9710279). The SOHO/MDI magnetogram used for the comparison is kindly provided by SOI-MDI group. The high voltage switchable power supply for $\mathrm{KD}^{*} \mathrm{P}$ modulator was designed and fabricated at the Space Application Center (SAC), Ahmedabad, for which we acknowledge the contribution of Dr. S.S. Rana, Mr. R.K. Arora and his group members. Dr. D. Balasubramaniam and Dr. Vyas from SAC are acknowledged for their help in optical coating. The mechanical components were fabricated by Mr. B.L. Paneri of USO.

\section{References}

Bonaccini D., Stauffer F., 1990, A\&A 229, 817

Canfield R.C., Beaujardiére J.F., Yuhong F., et al., 1993, ApJ 411,362

Cauzzi G., Smaldone L.A., Balasubramaniam K.S., Keil S.L., 1993, Sol. Phys. 146, 207

Debi Prasad C., Ambastha A., Ai G., 1998, Sol. Phys. 179, 13

Debi Prasad C., Mathew S.K., Bhatnagar A., Ambastha A., 1998, Exp. Astro. 8, 2

Heyvaerts J., Priest E.R., Rust D., 1977, ApJ 216, 123

Jefferies J.J., Mickey D.L., 1991, ApJ 372, 694

Mathew S.K., 1998, A study of Solar Magnetic and Velocity Fields, Ph.D Thesis

Meaburn J., 1970, Ap\&SS 9, 206

Rust D.M., Burton C.H., Leistner A.L., 1987, Soc. Photo-Opt. Inst. Eng. Conf. Proc. 627

Scherrer P.H., Bogart R.S., Bush R.I., et al., 1995, Sol. Phys. 162,129

Varsik R.C., 1995, Sol. Phys. 161, 207

Vaughan J.M., 1989, The Fabry-Perot interferometer, Adam Hilger, Bristol \& Philadelphia

West E.A., 1989, Soc. Photo-Opt. Inst. Eng. Conf. Proc. 1166, 434

Zirin H., 1995, Sol. Phys. 159, 203 